\title{
Interfacing a parallel simulation of a neuronal network to robotic hardware using MUSIC, with application to real-time figure-ground segregation
}

\author{
Ali Nazem ${ }^{1,2}$, Gert Kootstra ${ }^{1}$, Danica Kragic ${ }^{1}$, Mikael Djurfeldt2,3* \\ From Twentieth Annual Computational Neuroscience Meeting: CNS*2011 \\ Stockholm, Sweden. 23-28 July 2011
}

MUSIC, the multi-simulation coordinator, supports communication between neuronal-network simulators, or other (parallel) applications, running in a cluster super-computer [1,4]. Here, we've developed a MUSICenabled class library providing an interface between MUSIC-enabled applications and applications running on computers outside of the cluster. Specifically, we have used this component to interface the cameras of a robotic head to a neuronal-network simulation running on a Blue Gene/L supercomputer [2]. The interface enables the neuronal-network simulator to receive real- world images in real-time from the robot. Moreover, it enables the robot to be controlled by the neuronal network. The neuronal-network simulation implements a model of figure-ground segregation based on neuronal activity in the Macaque visual cortex [3].

I. A special purpose TCP/IP based communication interface has been implemented in $\mathrm{C}++$ as an extendible class library. The architecture of the interface is shown in Figure 1 . The client end of the interface is specifically designed to meet the requirements for operating on the Blue Gene /L, as well as being MUSIC-aware. The server

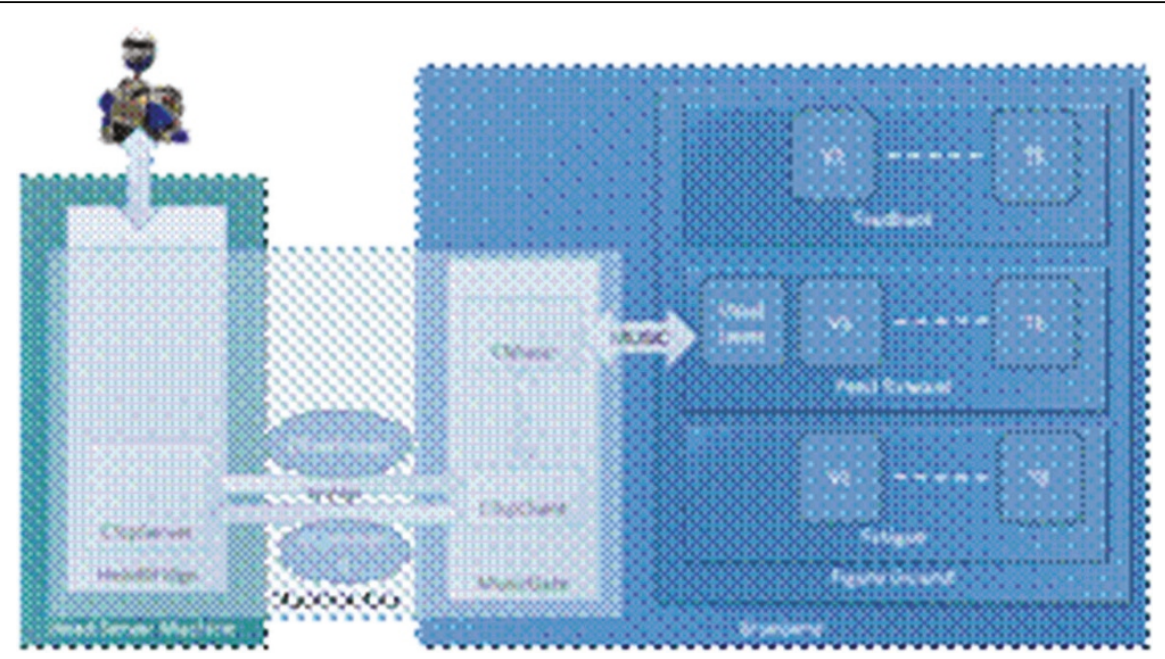

Figure 1 The MUSIC interface architecture.

\footnotetext{
* Correspondence: djurfeldt@incf.org

²PDC, CSC, KTH, 10044 Stockholm, Sweden

Full list of author information is available at the end of the article
}

(c) 2011 Nazem et al; licensee BioMed Central Ltd. This is an open access article distributed under the terms of the Creative Commons 
side component resides in the outside world providing the client with streaming real-time data. We designed an inheritable class called CSerializable that defines the unit of data and marshals the data across the route from the source to the final destination in the parallel application. Based on CSerializable, we implemented the entities CCommand and CRawImage which are required for transmission of control commands and images, respectively, between the parallel application and the robot (Figure 1). A single process application called MusicGate, connects the client side of the interface and the musicenabled component together. The parallel application sends a command to the server requesting a stream of images. As soon as one frame of the data sent from the server is available in MusicGate, it will be directly transferred from the read buffer to the parallel application by the MUSIC library, hence avoiding redundant internal memory operations. The implemented architecture performs the communication IO operations in parallel with the neural processing in order to minimize the idle time in compute nodes. The idle time is a function of communication latency, and the processing load of the neuronalnetwork simulation.

II. Having the communication interface, we implemented a parallel model for figure-ground segregation. The implementation covers the structure of neurons, neuronal layers, and neuronal networks that can operate on a parallel platform. We defined the concept of a tile of neurons. One dedicated processor is allocated to each tile of neurons. Each layer contains a number of tiles.

\section{Results}

We have successfully implemented an interface between a neuronal-network simulator running on a parallel computer and a robot in the real world. The latency and transfer rate of the entire model makes real-time figure-ground segregation possible. Since the client side of the interface provides a standard MUSIC port towards the parallel application, it can be used to connect the robotic head to a generic MUSIC-enabled neuronal-network simulator. We demonstrate this for the NEST simulator [4].

\section{Author details}

${ }^{1}$ CVAP, CSC, KTH, 10044 Stockholm, Sweden. ${ }^{2}$ PDC, CSC, KTH, 10044 Stockholm, Sweden. ${ }^{3}$ INCF, Karolinska Institutet, Nobels väg 15A, 17177 Stockholm, Sweden.

Published: 18 July 2011

\section{References}

1. Örjan Ekeberg, Mikael Djurfeldt: MUSIC - Multi-Simulation Coordinator User's Manual. 2009.

2. Mullen-Schultz Gary L: Blue Gene/L: Application Development. IBM 2006.
3. Roelfsema Pieter R, Lamme Victor AF, Henk Spekreijse, Holger Bosch: Figure-Ground Segregation in a Recurrent Network Architecture. Journal of Cognitive Neuroscience 2002, 14(4):525-537.

4. Mikael Djurfeldt, Johannes Hjorth, Eppler Jochen M, Niraj Dudani, Moritz Helias, Potjans Tobias C, Bhalla Upinder S, Markus Diesmann, Kotaleski Jeanette Hellgren, Örjan Ekeberg: Run-Time Interoperability Between Neuronal Network Simulators Based on the MUSIC Framework. Neuroinform 2010.

\section{doi:10.1186/1471-2202-12-S1-P78}

Cite this article as: Nazem et al:: Interfacing a parallel simulation of a neuronal network to robotic hardware using MUSIC, with application to real-time figure-ground segregation. BMC Neuroscience 2011 12(Suppl 1): P78.

\section{Submit your next manuscript to BioMed Central and take full advantage of:}

- Convenient online submission

- Thorough peer review

- No space constraints or color figure charges

- Immediate publication on acceptance

- Inclusion in PubMed, CAS, Scopus and Google Scholar

- Research which is freely available for redistribution

Submit your manuscript at www.biomedcentral.com/submit 